\title{
Contaminación atmosférica y enfermedades respiratorias en Cochabamba, Bolivia
}

\author{
Atmospheric pollution and respiratory diseases in Cochabamba, Bolivia
}

\author{
Contaminação atmosférica e doenças respiratórias em Cochabamba, Bolívia
}

\section{Nohemí Ventura Canaviri', Ismael Morales Ojeda', Ramón Carles Gelabert Santané3}

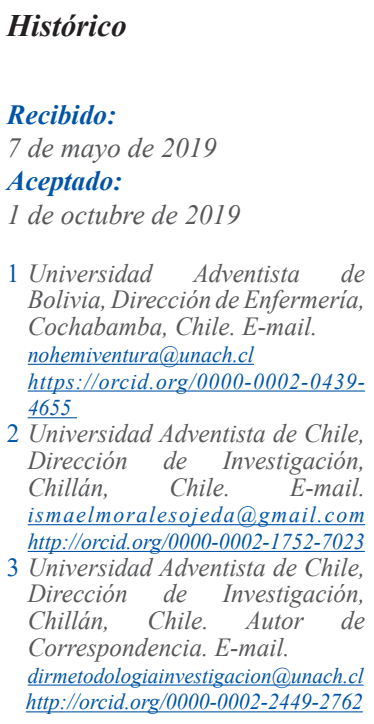

Introducción: La contaminación atmosférica es un problema medioambiental que afecta de manera negativa a la salud. Objetivo: Asociar los niveles de contaminación atmosférica y la morbilidad respiratoria. Materiales y Métodos: Estudio ecológico, observacional realizado a la población urbana de la ciudad de Cochabamba, del 1 de enero de 2012 al 31 de diciembre de 2016. Los datos se recolectaron a través de la Red de Monitoreo de la Calidad de Aire de Cochabamba (estaciones de Semapa, Coña Coña y Parque Kanata) y el Hospital Clínico Viedma. El análisis estadístico se realizó con la prueba de correlación de Spearman. Resultados: El promedio de casos semanales de enfermedades respiratorias presentó una correlación positiva muy baja con el promedio semanal de $\mathrm{PM}_{10}$ de las tres estaciones de monitoreo atmosférico $(\mathrm{r}=0,181, p<0,004 ; \mathrm{r}=0,242, p<0,001 ; \mathrm{r}=0,183, p<0,011) \mathrm{y}$ una correlación positiva baja o muy baja con el promedio semanal de $\mathrm{NO}_{2}$ registrada cada hora $(\mathrm{r}=0,365, p<0,000 ; \mathrm{r}=0,127, p<0,05)$. También de forma consistente se observó una correlación negativa baja con el promedio semanal de HR $(\mathrm{r}=-0,388, p<0,000) \mathrm{y}$ muy baja con el promedio semanal de precipitaciones $(\mathrm{r}=-0,250, p<0,000)$. Discusión y Conclusiones: Se observó una correlación lineal positiva baja o muy baja entre los ingresos semanales por enfermedades respiratorias y el promedio de contaminantes atmosféricos en el periodo 2012-2016.

Palabras clave: Contaminación del Aire; Enfermedades Respiratorias; Enfermedad Pulmonar Obstructiva Crónica; Material Particulado.

Abstract

Introduction: Atmospheric pollution is an environmental problem that negatively affects health. Objective: To associate the levels of atmospheric pollution with respiratory morbidity. Materials and Methods: An ecological observational study was conducted with the urban population of the city of Cochabamba from January $1^{\text {st }}, 2012$ to December $31^{\text {st }}, 2016$. Data was collected through the Cochabamba Air Quality Monitoring Network (Semapa, Coña Coña and Kanata Park stations) and the Viedma Clinical Hospital. The statistical analysis was performed using Spearman's rank correlation test. Results: The average number of weekly cases of respiratory diseases showed a very weak positive correlation with a weekly average of $\mathrm{PM}_{10}$ in the three atmospheric monitoring stations $(\mathrm{r}=0.181, p<0.004 ; \mathrm{r}=0.242, p<0.001 ; \mathrm{r}=0.183, p<0.011)$ and a weak or very weak positive correlation with a weekly average of $\mathrm{NO}_{2}$ recorded every hour $(\mathrm{r}=0.365, p<0.000 ; \mathrm{r}=0.127, p<0.05)$. A weak negative correlation with the weekly average of RH $(\mathrm{r}=-0.388, p<0.000)$ and a very weak correlation with the weekly average of precipitations $(\mathrm{r}=-0.250, p<0.000)$ was also consistently observed. Discussion and Conclusions: A weak or very weak positive linear correlation was observed between the weekly admissions due to respiratory diseases and the average of atmospheric pollutants over the period 2012-2016.

Key words: Air Pollution; Respiratory Tract Diseases; Pulmonary Disease, Chronic Obstructive; Particulate Matter.

Resumo

Introdução: A contaminação atmosférica é um problema meio ambiental que afeta negativamente à saúde. Objetivo: Associar os níveis de contaminação atmosférica e a morbilidade respiratória. Materiais e Métodos: Estudo ecológico, observacional realizado na população urbana da cidade de Cochabamba, de 1 de janeiro de 2012 à 31 de dezembro de 2016. Os dados foram coletados através da Rede de Monitoramento da Qualidade do Ar de Cochabamba (estações de Semapa, Coña Coña e Parque Kanata) e o Hospital Clínico Viedma. A análise estatística foi realizada através do teste de correlação de Spearman. Resultados: A média de casos semanais de doenças respiratórias apresentou uma correlação positiva muito baixa a respeito da média semanal de $\mathrm{PM}_{10}$ das três estacoes de monitoramento atmosférico $(\mathrm{r}=0,181, p<0,004 ; \mathrm{r}=0,242, p<0,001 ; \mathrm{r}=0,183, p<0,011)$ e uma correlação positiva baixa ou muito baixa a respeito da média semanal de $\mathrm{NO}_{2}$ registrada cada hora $(\mathrm{r}=0,365, p<0,000 ; \mathrm{r}=0,127, p<0,05)$. Verificou-se também de forma consistente uma correlação negativa baixa a respeito da média semanal de $\mathrm{HR}(\mathrm{r}=-0,388, p<0,000)$ e muito baixa com a média semanal de precipitações $(r=-0,250, p<0,000)$. Discussão e Conclusões: Observou-se uma correlação lineal positiva baixa ou muito baixa entre a renda semanal por doenças respiratórias e a média de contaminantes atmosféricos no período $2012-2016$. Palavras chave: Poluição do Ar; Doenças Respiratórias; Doença Pulmonar Obstrutiva Crônica; Material Particulado.

Como citar este artículo: Ventura NC, Morales IO, Gelabert RCS. Contaminación atmosférica y enfermedades respiratorias en Cochabamba, Bolivia. Rev Cuid. 2020; 11(1): e870. http://dx.doi.org/10.15649/cuidarte.870

(c) (1) () 2020 Universidad de Santander. Este es un artículo de acceso abierto, distribuido bajo los términos de la licencia Creative Commons Attribution (CC BY-NC 4.0), que permite el uso ilimitado, distribución y reproducción en cualquier medio, siempre que el autor original y la fuente sean debidamente citados. 


\section{INTRODUCCIÓN}

La vía aérea es una de las principales vías de acceso de los contaminantes atmosféricos al organismo. Según estimaciones de la Organización Mundial de la Salud (OMS), para 2008, se estima que el número de muertes prematuras atribuibles a la contaminación del aire exterior urbano asciende a 1,34 millones en todo el mundo. De estos, 1,09 millones de muertes podrían evitarse si se implementaran los valores medios anuales de las Directrices de calidad del aire de $\mathrm{PM}_{10}=20 \mu \mathrm{g}$ $/ \mathrm{m}^{3} \mathrm{y} \mathrm{PM}_{2.5}=10 \mu \mathrm{g} / \mathrm{m}^{3}$.

En 2012, alrededor de un $72 \%$ de las defunciones prematuras estuvieron relacionadas a la contaminación del aire exterior debido a causas como cardiopatía isquémica y accidente cerebrovascular. Mientras que un 14\% se debió a neumopatía obstructiva crónica o infección aguda de las vías respiratorias inferiores, así como un 14\% de las defunciones fue a causa de cáncer de pulmón².

En Bolivia, un estudio comparativo realizado en tres ciudades capitales del país con diferentes características climáticas y alturas sobre el nivel del mar, mostró que los niveles de $\mathrm{PM}_{10}$ fueron un factor de riesgo importante tanto para infecciones respiratorias agudas (IRAs) como para neumonías en las tres ciudades. Se observó que el riesgo era mayor en El Alto, ciudad ubicada a mayor altura, seguido por la Paz, y Cochabamba que presentaba el menor riesgo. Al mismo tiempo se observó que la amplitud térmica era también un factor de riesgoํㅡ. Si bien en Bolivia y en la ciudad de Cochabamba no existen investigaciones publicadas que evidencien la correlación entre la contaminación atmosférica urbana y las enfermedades respiratorias, es posible detectar el problema epidemiológico a partir de distintas fuentes. Un informe realizado por la OMS sobre la calidad del aire en 1.600 ciudades del mundo señala a Cochabamba como una de las urbes más contaminadas de la región²,3 ${ }^{2}$. En 2017 un neumólogo de la Caja Nacional de Salud (CNS) de la ciudad de Cochabamba, indicó que el $80 \%$ de los pacientes que atendía de manera diaria presenta problemas de asma.

La contaminación atmosférica en la ciudad de Cochabamba se mide principalmente por material particulado $\left(\mathrm{PM}_{10}\right)$, situación topográfica, parque automotor, industrias y actividades industriales como el cocimiento de ladrillos, festividades como la Noche de San Juan donde las personas encienden gran número de fogatas, incendios forestales, efecto chaqueos o quema de cultivos o malezas. La Fundación Suiza de Cooperación para el Desarrollo Técnico (SwissContact) en Cochabamba, ha destacado que el monóxido de carbono que producen los autos y los gases de los hidrocarburos mal combustionados son los principales contaminantes del aire en la urbe de Cochabamba $^{4,5}$. Por este motivo, es que se buscó investigar la correlación entre la contaminación atmosférica y los ingresos hospitalarios respiratorios.

\section{MATERIALES Y MÉTODOS}

Se realizó un estudio ecológico, en el que se recogieron los datos de exposición de las variaciones temporales en los niveles de contaminación atmosféricos por $\mathrm{PM}_{10}$ y se correlacionó con los 
cambios en la frecuencia del número de atenciones hospitalarias de urgencia y de consulta externa por morbilidad respiratoria.

Los datos sobre las visitas hospitalarias a los servicios de urgencias y de consulta externa (desde enero de 2012 hasta diciembre de 2016) se obtuvieron de las bases de datos del Sistema Nacional de Información en Salud correspondientes al Hospital Clínico Viedma de la ciudad de Cochabamba, Bolivia que recopilaron las siguientes variables de cada paciente atendido: edad, sexo, fecha de visita y el diagnóstico de alta, además del diagnóstico principal de atención mediante la Clasificación Internacional de Enfermedades (CIE) en su revisión $N^{o} 10$. En este estudio se seleccionaron los pacientes diagnosticados con los siguientes códigos de enfermedades respiratorias crónicas (CIE-10: J40-J46): bronquitis no especificada como aguda o crónica (J40), bronquitis crónica simple y mucopurulenta (J41), bronquitis crónica sin especificar (J42), enfisema (J43), otras enfermedades pulmonares obstructivas crónicas (J44), asma (J45) y estado asmático (J46).

Las concentraciones diarias de material particulado con diámetro aerodinámico menor o igual a 10 micras $\left(\mathrm{PM}_{10}\right)$, dióxido de Azufre $\left(\mathrm{SO}_{2}\right)$, dióxido de nitrógeno $\left(\mathrm{NO}_{2}\right)$, ozono $\left(\mathrm{O}_{3}\right)$, temperatura, humedad relativa (HR), velocidad y dirección del viento, se recogieron de las mediciones efectuadas del sistema de seguimiento ambiental automatizado proporcionado por la Red de Monitoreo de la Calidad del Aire (Red MoniCA) de la ciudad de Cochabamba de los periodos 20122016.
El sistema automático de la Red MoniCA actualmente cuenta con tres estaciones de monitoreo atmosférico, una ubicada en la parte norte de la ciudad (predios de Semapa), otra en el sector sur de la ciudad (predios del Parque Kanata) y la última en el sector oeste de la ciudad (predios de Coña Coña). En la estación de monitoreo de Semapa, se miden los parámetros de $\mathrm{PM}_{10}, \mathrm{O}_{3}$ y óxidos de nitrógeno $\left(\mathrm{NO}\right.$ y $\mathrm{NO}_{2}$ ), además se cuenta con la medición de variables meteorológicas como velocidad del viento, dirección del viento, temperatura, humedad relativa, radiación solar y precipitación. La estación de monitoreo de Parque Kanata es bastante completa por la medición de gases y partículas, esta tiene analizadores ambientales que miden $\mathrm{O}_{3}, \mathrm{SO}_{2}, \mathrm{NO}, \mathrm{NO}_{2}$ y $\mathrm{PM}_{10}$. La estación de monitoreo de Coña Coña permite la medición de $\mathrm{PM}_{10} \mathrm{y}$ variables meteorológicas como velocidad del viento, dirección del viento, temperatura y radiación solar. Los equipos son programados para proporcionar datos cada1 $5 \mathrm{mi}-$ nutos, las 24 horas del día y los 365 días del año. La recolección de los datos se realiza vía internet, a través del servidor de la unidad de sistemas del municipio de Cochabamba.

A partir de las bases de datos, se resumió la información obtenida en promedios de cada variable, distribuidos en 258 semanas (periodos 20122016). Se utilizó el programa SPSS.v19 para realizar la prueba de correlación de Spearman y determinar la relación entre las concentraciones diarias de contaminantes atmosféricos y el número de visitas a los servicios de urgencias y de consulta externa por enfermedades respiratorias. 


\section{RESULTADOS}

En el período entre 2012 y2016 se diagnosticaron un promedio de 50 casos semanales de enferme- dad respiratoria, de los que en promedio aproximadamente el $7 \%(n=4)$ fueron casos de enfermedad pulmonar obstructiva crónica (EPOC) y el 12\% $(n=6)$ de asma (ver Tabla 1$)$.

Tabla 1. Casos de enfermedad respiratoria en el Hospital Clínico Viedma, Cochabamba, Bolivia, 2012 a 2016

\begin{tabular}{lccccc} 
& & \multicolumn{4}{c}{ Casos semanales } \\
\cline { 3 - 6 } & $\begin{array}{r}\text { Semanas con registro } \\
\text { de casos 2012-2016 }\end{array}$ & Mínimo & Máximo & Media & Desv. \\
típ.
\end{tabular}

Fuente: Elaboración propia

El promedio de casos semanales de enfermedades respiratorias que acudieron a consultas de urgencia o especialidad presentó una correlación positiva muy baja con el promedio semanal de $\mathrm{PM}_{10}$ de las tres estaciones de monitoreo atmosférico $(\mathrm{r}=0,181, p<0,004 ; \mathrm{r}=0,242, p<0,001$; $\mathrm{r}=0,183, p<0,011)$ y una correlación positiva baja o muy baja con el promedio semanal de $\mathrm{NO}_{2}$ registrado cada hora para dos estaciones $(\mathrm{r}=$ $0,365, p<0,000 ; r=0,127, p<0,049)$, esto indica que hay una leve tendencia a un mayor número de casos de enfermedades respiratorias. También de forma consistente se observó una correlación negativa baja con el promedio semanal de HR (r $=-0,388, p<0,000)$ y muy baja con el promedio semanal de precipitaciones $(\mathrm{r}=-0,250, p<0,000)$ (Figura 1). La dirección del viento (WD) mostró una correlación consistente con el número de casos de enfermedades respiratorias, dado que en la estación meteorológica de Semapa presentó una correlación positiva muy baja y en cambio en la estación de Coña Coña la correlación fue negativa muy baja (Tabla 2). 
Tabla 2. Correlación de Spearman entre el total de casos respiratorios en urgencia y especialidad y los promedios de NO2 según las horas

\begin{tabular}{|c|c|c|c|}
\hline $\begin{array}{l}\text { Total de casos respiratorios } \\
\text { semanales en consultorio de } \\
\text { urgencia y especialidad }\end{array}$ & $\begin{array}{c}\text { Promedio de } \\
\text { Semapa } \mathrm{NO}_{2}(1 \mathrm{~h})\end{array}$ & $\begin{array}{l}\text { Promedio de } \\
\text { Semapa } \mathrm{NO}_{2} \\
\qquad(24 \mathrm{~h})\end{array}$ & $\begin{array}{l}\text { Promedio de } \\
\text { Parque Kanata } \\
\quad \mathrm{NO}_{2}(\mathbf{1} \text { h) }\end{array}$ \\
\hline Coeficiente de correlación & $0,365^{* *}$ & $0,213^{* *}$ & $0,127^{*}$ \\
\hline Sig. (bilateral) & 0,000 & 0,001 & 0,049 \\
\hline \multirow[t]{2}{*}{$\mathrm{N}$} & 233 & 235 & 243 \\
\hline & $\begin{array}{l}\text { Promedio } \mathrm{PM}_{10} \text { de } \\
\text { Parque Kanata }\end{array}$ & $\begin{array}{c}\text { Promedio de } \\
\mathrm{PM}_{10} \text { Coña Coña }\end{array}$ & $\begin{array}{c}\text { Promedio de } \mathrm{PM}_{10} \\
\text { Semapa }\end{array}$ \\
\hline Coeficiente de correlación & $0,181 * *$ & $0,242^{* *}$ & $0,183^{*}$ \\
\hline Sig. (bilateral) & 0,004 & 0,001 & 0,011 \\
\hline \multirow[t]{2}{*}{$\mathrm{N}$} & 258 & 203 & 192 \\
\hline & $\begin{array}{c}\text { Promedio de Semapa } \\
\text { WD }\end{array}$ & $\begin{array}{c}\text { Promedio de } \\
\text { Coña Coña WD }\end{array}$ & $\begin{array}{l}\text { Promedio de } \\
\text { Semapa HR }\end{array}$ \\
\hline Coeficiente de correlación & $0,206 * *$ & $-0,223^{* *}$ & $-0,388^{* *}$ \\
\hline Sig. (bilateral) & 0,006 & 0,003 & 0,000 \\
\hline \multirow[t]{2}{*}{$\mathrm{N}$} & 179 & 172 & 259 \\
\hline & $\begin{array}{l}\text { Promedio de Coña } \\
\text { Coña }{ }^{\circ} \mathrm{C}\end{array}$ & $\begin{array}{l}\text { Promedio de } \\
\text { Semapa precip }\end{array}$ & \\
\hline Coeficiente de correlación & $-0,157^{*}$ & $-0,250^{* *}$ & \\
\hline Sig. (bilateral) & 0,025 & 0,000 & \\
\hline $\mathrm{N}$ & 203 & 259 & \\
\hline
\end{tabular}

Fuente: Elaboración propia.

El total de casos semanales de enfermedades respiratorias presentó una correlación positiva muy baja con el promedio semanal de $\mathrm{SO}_{2}$ en $24 \mathrm{~h}$ registrado en Parque Kanata $(\mathrm{r}=0,247, p<0,00)$, es decir, que a mayores niveles de $\mathrm{SO}_{2}$ hay una leve tendencia al aumento de casos de enfermedades respiratorias (Figura 2).
La prueba de correlación no demostró relación entre el total de casos respiratorios semanales y los niveles de $\mathrm{O}_{3}$ por hora y en 24 horas en las estaciones Semapa y Parque Kanata (Figura 2). 


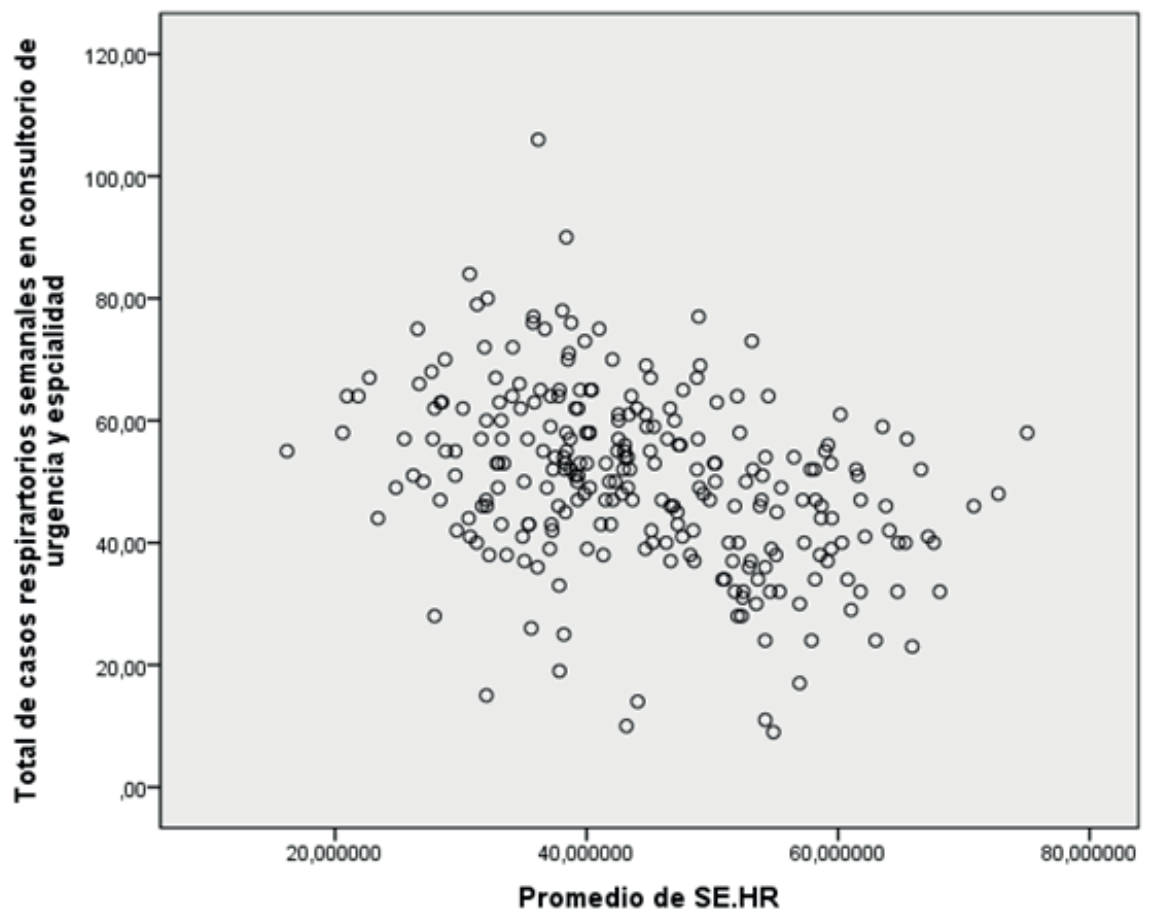

Figura 1. Correlación del total de casos semanales de enfermedades respiratorias y HR de Semapa

$\mathrm{SE}=$ Semapa; HR: humedad relativa.

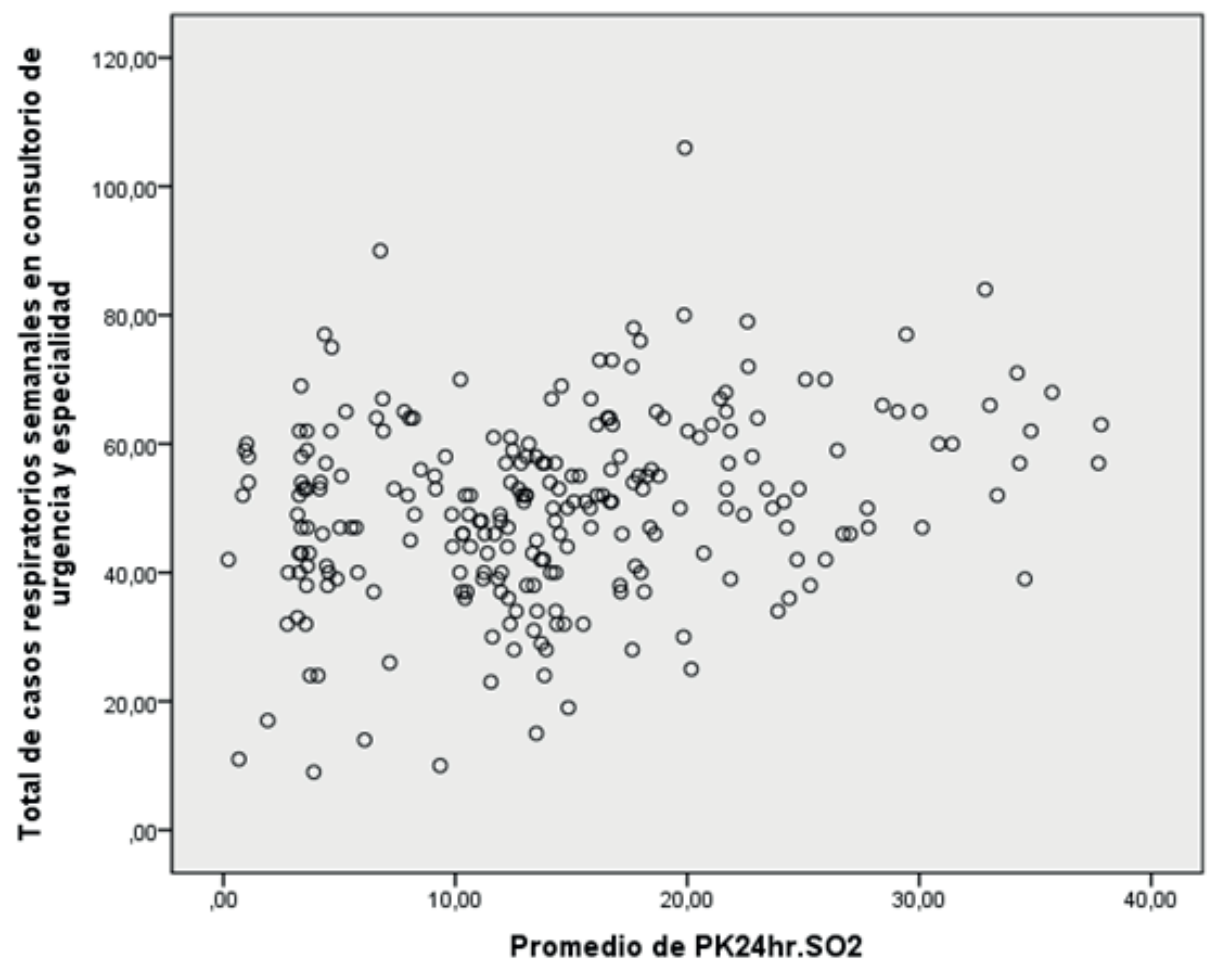

Figura 2. Correlación del total de casos semanales de enfermedades respiratorias y $\mathrm{SO}_{2}$ de 24 h de Parque Kanata. 
Al realizar un filtro de las enfermedades respi- do en la estación de Coña Coña con una correratorias, se observó que los casos semanales de lación positiva muy baja para EPOC ( $\mathrm{r}=0,170$; EPOC y asma -a diferencia de los demás- no $p<0,015)$, es decir, a mayores niveles de $\mathrm{PM}_{10}$ presentaron una correlación con los promedios podría haber una leve relación con un mayor núsemanales de $\mathrm{PM}_{10}$, a excepción de lo registra- mero de casos de EPOC (Tabla 3).

\section{Tabla 3. Casos de EPOC y asma según promedio semanal de $P M_{10}$ en las tres estaciones me- teorológicas de Cochabamba, años 2012-2016}

\begin{tabular}{|c|c|c|c|c|}
\hline \multirow{2}{*}{ Rho de Spearman } & & PK & $\mathrm{CC}$ & SE \\
\hline & & de. $\mathrm{PM}_{10}$ & de $\mathrm{PM}_{10}$ & de $\mathrm{PM}_{10}$ \\
\hline \multirow{3}{*}{$\begin{array}{l}\text { Casos semanales de } \\
\text { EPOC }\end{array}$} & Coeficiente de correlación &, 062 &, $170^{*}$ &, 123 \\
\hline & Sig. (bilateral) &, 325 & 015 & ,090 \\
\hline & $\mathrm{N}$ & 258 & 203 & 192 \\
\hline \multirow{3}{*}{$\begin{array}{l}\text { Casos semanales de } \\
\text { asma }\end{array}$} & Coeficiente de correlación &,- 087 &, 000 &,- 064 \\
\hline & Sig. (bilateral) & , 164 & ,999 &, 381 \\
\hline & $\mathrm{N}$ & 258 & 203 & 192 \\
\hline
\end{tabular}

$\mathrm{SE}=$ Semapa $\mathrm{CC}=$ Coña Coña $\mathrm{PK}=$ Parque Kanata $\mathrm{PM}_{10}=$ Material particulado (Elaboración propia) .

\section{DISCUSION}

Tal como se expresa en la 68 Asamblea Mundial de la Salud, cada año se producen 4,3 millones de defunciones por exposición a la contaminación doméstica del aire, a las que deben sumarse 3,7 millones de muertes al año asociadas a la contaminación atmosférica ${ }^{\underline{5}}$. En la actualidad la contaminación del aire es considerada una inequidad en salud que afecta principalmente a mujeres, niños y adultos mayores, de entre los que se destacan personas con bajos ingresos que están expuestas debido a los medios de calefacción y cocción de alimentos que disponen ${ }^{6}$. En el caso particular de Bolivia, la concentración media anual $\mathrm{PM}_{10}$ es de $27 \mathrm{~g} / \mathrm{m}^{3}$ en áreas rurales con una máxima de 45 , mientras que en áreas urbanas es de 32 con una máxima de 497. Particularmente en el caso de La Paz la medición de $\mathrm{PM}_{10}$ alcanzó niveles cercanos a $100 \mathrm{~g} / \mathrm{m}^{3}, 7$, niveles superiores a los encontrados en Bogotá, Cuidad de México y Sao Paulo ${ }^{7}$. Niveles similares a los alcanzados en la medición de $\mathrm{PM}_{10}$ en la estación Kanata.

En el caso de la población de Cochabamba se observó que existe también una baja correlación ( $\mathrm{r}$ $=0,2-0,4)$ entre los casos de enfermedad respiratoria y los contaminantes ambientales como $\mathrm{NO}_{2}$ 
y $\mathrm{PM}_{10}$ en las distintas estaciones (Tabla 3). Esto presentó coherencia con lo observado en el trabajo realizado en Busan, que cuenta sobre como aumentó el efecto de las PM sobre las enfermedades respiratorias a medida que disminuía la $\mathrm{HR}^{8} \stackrel{8}{\text {. }}$

Respecto a la polución ambiental del aire en Bolivia, en 2012 se atribuyeron 2.521 muertes, lo que corresponde a 25 muertes por cada 100.000 personas, de las cuales 265 fueron por enfermedad respiratoria baja aguda incluyendo a los niños menores de 5 años, 68 por EPOC, 88 por cáncer del pulmón, 1.126 por isquemia cardiaca ${ }^{7}$. Pese a lo alarmante de esta situación, en julio de 2017 el Servicio Departamental de Salud (SEDES) de la ciudad de Cochabamba a través del responsable de Vigilancia Epidemiológica, manifestó que no contaban con estudios específicos de contaminación, sino únicamente con estimados de prevalencia de enfermedades; además indicó que las IRAs podrían ser consecuencia de la contaminación atmosférica, sin embargo, afirmaba que el aire solamente es uno de muchos factores que pueden influir en la patología, reconoció que también puede afectar a grupos de riesgo, es decir personas con cardiopatías que necesitan oxigenación ${ }^{9}$. En concordancia con esta situación, esta investigación expone que el Hospital Clínico Viedma de la ciudad de Cochabamba atiende una media de 50 casos de ingresos semanales por enfermedades respiratorias que podrían relacionarse con la contaminación atmosférica. Situación similar a la ocurrida por ejemplo en Busan, Corea $^{9}$, donde entre 2007 y 2010 se concluyó que los niveles de PM y los factores meteorológicos afectaron la hospitalización por enfermedades respiratorias, especialmente en niños y adultos mayores.
Un estudio en Beijing, China, en 2014-2015, mostró que la contaminación atmosférica por partículas $\left(\mathrm{SO}_{2}, \mathrm{O}_{3}\right.$ y $\left.\mathrm{NO}_{2}\right)$ se asocia significativamente con las admisiones hospitalarias de enfermedad pulmonar obstructiva crónica. La susceptibilidad a la contaminación por partículas varió según el sexo y la edad ${ }^{10}$. Similar a lo que ocurre en Jinan, China donde se estudió la relación entre la contaminación del aire y visitas a las salas de emergencia para las enfermedades respiratorias (neumonía, asma, infección del tracto respiratorio superior y EPOC) en las zonas urbanas y suburbanas de Jinan, estas se asociaron significativamente con los niveles de contaminantes atmosféricos ( $\mathrm{smog}, \mathrm{SO}_{2}$ y $\mathrm{NO}_{2}$ ) y otros corroboraron que la visita en sala de urgencias para las enfermedades respiratorias puede ser utilizado como un indicador sensible de los resultados de salud de la contaminación del aire. Se encontró que el sexo femenino era más susceptible a la contaminación del aire que los hombres en la zona urbana y se observó la tendencia inversa en la zona suburbana ${ }^{11}$.

\section{CONCLUSIONES}

En el presente estudio hay una correlación baja entre los ingresos semanales por EPOC y el promedio de $\mathrm{PM}_{10}$ entre los años 2012-2016, lo que puede deberse a infraconsulta e infradiagnóstico asociado a la falta de recursos y excesiva distancia del servicio asistencial. En contraste, un estudio anterior realizado en Cochabamba encontró una asociación significativa entre vivir o estudiar en la zona Norte de la ciudad con sintomatología de asma, rinitis alérgica y eczema. En caso del asma, así como en este trabajo, Se estableció que 
vivir en la zona Norte, era un factor de riesgo para asma en relación a la zona Sur de la ciudad ${ }^{12}$.

Considerando los factores ambientales y sanitarios de la cuidad, es recomendable mejorar la vigilancia en las estaciones meteorológicas, pues se evidencian largos periodos donde no se realizan mediciones. Asimismo, es necesario reforzar el diagnóstico de enfermedades respiratorias, pues debido a la concentración demográfica y los niveles de $\mathrm{PM}_{10}$ es posible que exista un subdiagnóstico de los casos. Finalmente, debido a la alta confluencia de población indígena de distintas etnias se requiere concientización de la población acerca del uso de cocinas y calefacciones a base de combustibles sólidos (leña), junto con una campaña de incentivos para uso delos servicios de salud que permitan prevenir el daño pulmonar y cardíaco asociado a los niveles de contaminantes atmosféricos.

\section{Conflicto de intereses: Los autores declaran no} tener conflicto de intereses.

\section{REFERENCIAS}

1. World Health Organization. Environmental and social determinants of health (PHE) Burden of disease associated with urban outdoor air pollution for 2008. Disponible en: https://www.who.int/phe/health topics/outdoorair/databases/burden disease/en/

2. Luján M, Gonzáles D. Determinación del impacto de varios contaminantes criterio sobre la salud de la población en ciudades capitales de Bolivia. Acta Nova. 2016;7(3): 303-33.

3. World Health Organization. Public health, environmental and social determinants of health (PHE). Database: outdoor air pollution in cities. Disponible en: https:// www.who.int/phe/health_topics/outdoorair/databases/cities-2011/en/

4. Bascopé D. La Red de Monitoreo de la Calidad del Aire de Cochabamba (Red MoniCA). Acta Nova. 2003; 2(3): 282-91.
5. Armaza A, Ayo X, Céspedes R, Vargas I, Ortuño C, Luján M. Evaluación del efecto de Dióxido de Nitrógeno y Ozono en la salud de la población del Municipio Cercado. Cochabamba, Bolivia 2005-2006. Cochabamba, Bolivia: Agencia Suiza para el Desarrollo y la Cooperación - COSUDE, Fundación Suiza de Cooperación para el Desarrollo Técnico - Swisscontact, 2006. Disponible en: http://www.swisscontact.bo/sw_files/mlqorqmjggq.pdf

6. 68. a Asamblea mundial de la salud. Salud y medio ambiente: impacto sanitario de la contaminación del aire. Disponible en: http://apps.who.int/gb/ebwha/pdf files/WHA68/A68 R8sp.pdf

7. World Health Organization. Ambient air pollution: A global assessment of exposure and burden of disease. Ginebra: WHO Document Production Services, 2016. Disponible en: https://www.who.int/phe/publications/airpollution-global-assessment/en/

8. Jo EJ, Lee WS, Jo HY, Kim CH, Eom JS, Mok JH et al. Effects of particulate matter on respiratory disease and the impact of meteorological factors in Busan, Korea. Respir Med. 2017;124: 79-87. https://doi.org/10.1016/j.rmed.2017.02.010

9. Burgos CG. Cochabamba, una ciudad jardín envuelta en aire contaminado. Los Tiempos Digital. 2017.

10. Cao Y, Liu H, Zhang J, Huang KW, Zhao HY, Yang Y et al. Effect of particulate air pollution on hospital admissions for acute exacerbation of chronic obstructive pulmonary disease in Beijing. Beijing Da Xue Xue Bao Yi Xue Ban. 2017;49(3): 403-8.

11. Liu P, Wang X, Fan J, Xiao W Wang Y. Effects of Air Pollution on Hospital Emergency Room Visits for Respiratory Diseases: Urban-Suburban Differences in Eastern China. Int. J. Environ. Res. 2016;13(3). pii: E341. https://doi.org/10.3390/ijerph13030341

12. Cardozo L. Enfermedades alérgicas y contaminación atmosférica, en la población infantil-adolescente de Cochabamba, y análisis comparativo de prevalencias en zonas con diferente grado de exposición. (MP10, O3 Y NO2) [Tesis de maestría]. Bolivia: Universidad Mayor de San Simón; 2017. 